\title{
Enhancing I rregular Repetition Slotted ALOHA with Polarization Diversity in LEO Satellite Networks
}

\author{
Jingrui Su ${ }^{1}$, Guangliang Ren ${ }^{1 *}$, Bo Zhao ${ }^{1}$, and Jian Ding ${ }^{1,2}$ \\ ${ }^{1}$ The State Key Laboratory of Integrated Service Networks, Xidian University, \\ Xi'an 710071, China \\ ${ }^{2}$ National Key Laboratory of Electromagnetic Environment, China Research Institute of Radiowave Propagation, \\ Qingdao, 266107, China \\ [e-mail: sujingrui4213@126.com; glren@mail.xidian.edu.cn; zhaobo19920305@126.com] \\ [e-mail: dj2020@126.com] \\ *Corresponding author: Guangliang Ren
}

Received June 1, 2020; revised July 16, 2020; accepted August 10, 2020;

published September 30, 2020

\begin{abstract}
An enhanced irregular repetition slotted ALOHA (IRSA) protocol is proposed by using polarization characteristic of satellite link and MIMO detection in low earth orbit (LEO) satellite networks, which is dubbed polarized MIMO IRSA (PM-IRSA). In the proposed scheme, one or two packets in one slot can be decoded by employing polarized MIMO detection, and more than two collided packets in multiple slots which can construct the virtual MIMO model can be decoded by the MIMO detection algorithm. The performance of the proposed scheme is analyzed with the density evolution (DE) approach and the degree distribution is optimized to maximize the system throughput by using a differential evolution. Numerical results certify our analysis and show that the normalized throughput of the proposed PM-IRSA can achieve 1.89 bits/symbol.
\end{abstract}

Keywords: Random access, IRSA, satellite networks, Internet of Things (IoT), polarization diversity, virtual MIMO.

This work was supported in part by the National Natural Science Foundation of China under Grant No.91538105 and No.61801352. 


\section{Introduction}

IoT-oriented satellite network is attracting a lot of attention [1], especially in the context of the fifth generation mobile communication. In IoT-oriented low earth orbit (LEO) satellite networks, a large number of satellite machine-type terminals (SMTs) are noncooperative, which makes the traditional TDMA, MF-TDMA, and DAMA satellite multiple access protocols very inefficient. However, random access (RA) can be a promising solution for the satellite network with massive terminals.

Over the past decades, many RA protocols have been proposed to enhance the system performance [2]-[4]. A typical RA protocol named contention resolution diversity slotted ALOHA (CRDSA) is proposed in [2], where each packet is sent twice in randomly selected slots and an iterative interference cancellation (IC) technique is utilized to decode the overlapped packets. As an enhanced version of CRDSA, CRDSA++ is proposed in [3], where more than two replicas are transmitted and the maximum normalized throughput can reach about 0.68 packets/slot. However, as the load increases, the number of unresolved collision patterns in or among slots increases, the throughput of RA system with CRDSA++ is greatly limited.

To improve the throughput of RA system, literature [4] proposed irregular repetition slotted ALOHA (IRSA) by choosing mutable packet duplication rates in accordance with a certain probability, and viewed IC process as bipartite graph which can be utilized depict the structure of iterative codes to decode collision packets. The maximum normalized throughput of IRSA can reach 0.97 packets/slot for large frames. But as the load increases, unresolved collision patterns still limit the throughput improvement of RA with IRSA.

To further improve the throughput of IRSA, literature [5] proposed a power domain NOMA-IRSA to reduce the number of unresolved collision patterns. In this protocol, packets of each SMT can be transmitted with different power levels, and degree distributions with different number of power levels are optimized. As expected, the throughput is greatly improved, but the energy dissipation of terminals is also highly increased, especially for satellite reverse link with a large path loss. For SMTs with the limited battery life and transmitted power, the number of power levels are limited, which greatly reduced the throughput improvement of NOMA-IRSA. Moreover, more advanced RA protocols related to CRDSA and IRSA, such as generalized encoding CRDSA, enhancing the physical layer of CRDSA, IRSA with the capture effect, are proposed in [6]-[8] respectively, which among these advanced protocols utilized the capture effect. Although the throughput is improved, the energy consumption is also increased due to a larger transmit power required, which poses huge challenges in practical applications. Moreover, Q-learning based IRSA for wireless sensor networks (WSNs) is proposed in [9]. The scheme provides insights into RA scheme design, but it is not suitable for satellite networks due to the large delay caused by online learning.

The previous improvement schemes almost all focus on the decoding in one slot on single antenna and neglect some characteristics of the satellite channel, such as the polarization characteristic, so the improvement in throughputs of these schemes is limited. As the number of IoT devices increases rapidly, it is required to design a new random access scheme with a higher throughput by making full use of characteristics of the satellite channel. In this paper, we propose an enhanced IRSA to further improve the throughput of RA systems by employing polarization characteristics of satellite channel, which provides an additional freedom for the 
data transmission in the terrestrial satellite link (TSL). We utilize this new physical (PHY) layer feature and multiple-input multiple output (MIMO) detection in satellite RA network, and propose a new RA scheme, which is dubbed as polarization MIMO IRSA(PM-IRSA). The main contributions of this paper are summarized as follows.

1) To further improve the throughput of RA scheme, we propose a novel polarization diversity based IRSA RA scheme in IoT-oriented satellite networks. By considering polarization characteristics of satellite channel, receiver can take full advantage of this freedom degree so that two and more packets can be successfully decoded by dual-polarization MIMO detector and virtual MIMO detection algorithms, respectively.

2) We provide the theoretical analysis framework to evaluate the performance of the proposed scheme. The performance of PM-IRSA protocol is analyzed by using density evolution (DE) recursion.

3) With the proposed scheme, the structure of bipartite graph in PM-IRSA is changed, and degree distribution is optimized to maximize the throughput of the proposed protocol. We also numerically show it outperforms traditional RA schemes in terms of normalized throughput and PLR.

In Section 2, we present the system model. The proposed random access scheme is proposed in Section 3. Section 4 is density evolution analysis and degree distribution optimization about PM-IRSA. Numerical results are given in Section 5. Finally, in Section 6, the conclusion is given.

\section{System Model}

\subsection{Preliminaries}

We consider an IoT-oriented satellite network with the polarization transmission technique, where SMTs transmit burst packets to a satellite node. Each SMT is equipped with a linear cross polarization (LP) antenna, and the satellite node is equipped with a horizontal polarization (HP) and a vertical polarization (VP) antennas to receive the VP and the HP signals, respectively. Note that the VP and HP signals are processed with the same carrier oscillator and clock, thus, the polarization synchronization can be assured. The network model is shown in Fig. 1. The satellite sends on the data packets of SMTs to the gateway station, which execute the packet detection and decoding. In this case, the large transmission latency is an inherent feature. The number of activated users in a frame is a Poisson random variable, $\lambda=N G G_{p g}$ denotes the average packet arrival rate, where $N, G$ and $G_{p g}=1 /\left(R \cdot \log _{2} O\right)$ denote the number of time slots in a frame, the load and processing gain. $R$ is code rate, and $O$ is modulation cardinality. The duration of one frame is assumed to be $T_{F}$, so duration of one slot is give as $T_{S}=T_{F} / N$. We assume that all SMTs are slot- and frame-synchronous. Compared with existing traditional geostationary earth orbit (GEO) and medium earth orbit (MEO) satellite network, LEO satellite communications have smaller propagation delay, path loss and transmission power, and have flexible elevation angles. In addition, LEO satellite communications have richer reflection and scattering components and smaller LOS component compared to GEO and MEO satellite network, it can ensure the uncorrelated characteristics of MIMO channels. 


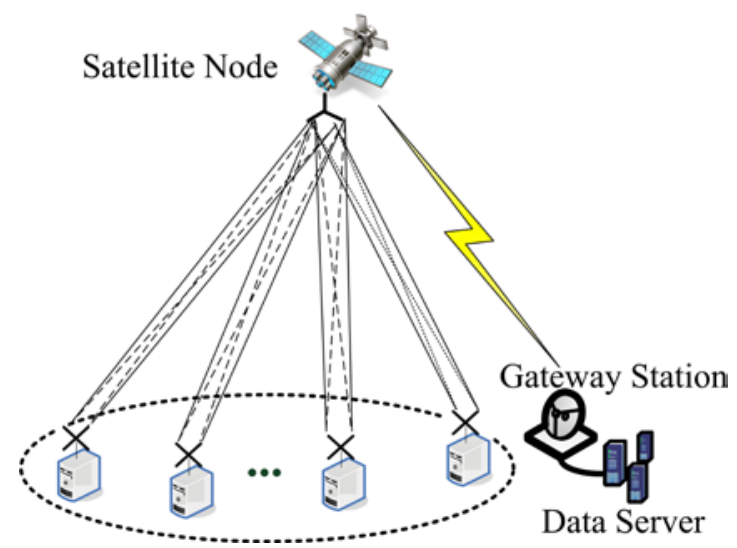

Fig. 1. IoT-oriented satellite network model

\subsection{Signal Model}

The single-carrier interleave frequency division multiple access (SC-IFDMA) signal with constant envelope characteristics is used to transmit the data packets from the SMTs. Each burst includes preamble, payload symbols, postamble, and pilot are uniformly inserted into data symbols. The structure os the packet is shown as Fig. 2.

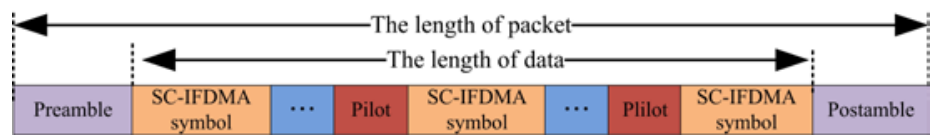

Fig. 2. Packet structure based on SC-IFDMA.

If a packet generated by the $u^{\text {th }}$ SMT in the $i^{\text {th }}$ slot can be represented as $\mathbf{x}_{i}^{(u)}=\left[x_{i}^{(u)}(1), \ldots, x_{i}^{(u)}(k), \ldots, X_{i}^{(u)}\left(L^{R A}\right)\right]$, where $L^{R A}$ is the number of the symbols in a packet, and $x_{i}^{(u)}(k)$ is complex vector of a symbol. If the packet is transmitted in the $i^{\text {th }}$ slot in a frame, and the polarization channel model from the $u^{\text {th }}$ SMT to the satellite node can be represented as

$$
\mathbf{h}_{i}^{(u)}=\left[\begin{array}{l}
h_{i, H}^{(u)} \\
h_{i, V}^{(u)}
\end{array}\right]=\left[\begin{array}{l}
h_{i, H H}^{(u)}+h_{i, H V}^{(u)} \\
h_{i, V V}^{(u)}+h_{i, V H}^{(u)}
\end{array}\right],
$$

where $h_{i, H}^{(u)}=h_{i, H H}^{(u)}+h_{i, H V}^{(u)}$ and $h_{i, V}^{(u)}=h_{i, V V}^{(u)}+h_{i, V H}^{(u)} \cdot h_{i, V V}^{(u)}$ and $h_{i, H H}^{(u)}$ denote the co-polarization channel impulse responses (CIR) between the VP/HP SMT and VP/HP satellite respectively, $h_{i, V H}^{(u)}$ and $h_{i, H V}^{(u)}$ are the cross-polarization CIR between the VP/HP SMT and HP/VP satellite respectively. The received signal in the $i^{\text {th }}$ slot from the $u^{\text {th }}$ activated SMTs can be represented as

$$
\mathbf{y}_{i}^{(u)}=\left[\begin{array}{l}
\mathbf{y}_{i, H}^{(u)} \\
\mathbf{y}_{i, V}^{(u)}
\end{array}\right]=\left[\begin{array}{cc}
h_{i, H H}^{(u)} & h_{i, H V}^{(u)} \\
h_{i, H V}^{(u)} & h_{i, H H}^{(u)}
\end{array}\right]\left[\begin{array}{l}
\mathbf{x}_{i}^{(u)} \\
\mathbf{x}_{i}^{(u)}
\end{array}\right]+\left[\begin{array}{l}
\mathbf{n}_{i, H} \\
\mathbf{n}_{i, V}
\end{array}\right]
$$

where $\mathbf{y}_{i, V}^{(u)}$ and $\mathbf{y}_{i, H}^{(u)}$ are the signals received by the VP and HP antennas respectively. $\mathbf{n}_{i, V}$ and $\mathbf{n}_{i, H}$ represents AWGN vector. It is assumed that $K$ activated SMTs transmit their packets 
simultaneously in the given $i^{\text {th }}$ slot, which can be represented as

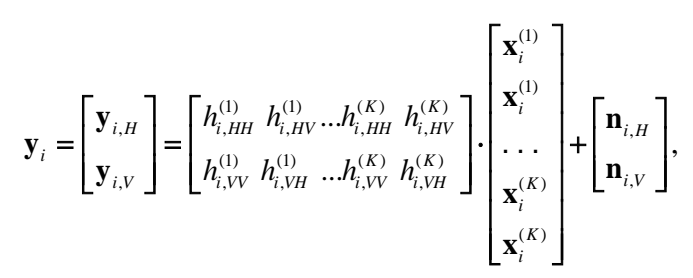

If packets of $M$ SMTs are distributed on $J$ slots, $j_{1}, j_{2}, \ldots, j_{J}$ and $u_{1}, u_{2}, \ldots, u_{M}$ denote the indexes of slots and SMTs respectively, the received signals in the $J$ slots can be represented as a multi-slots multi-SMTs polarized MIMO transmission model,

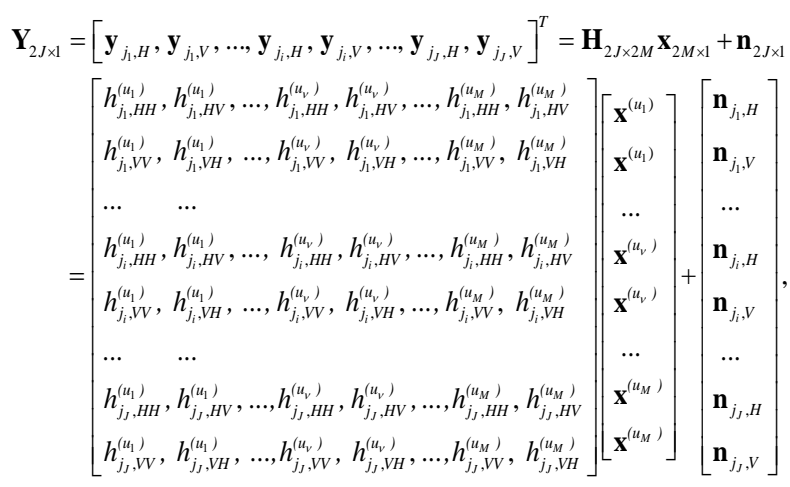

where the $h_{j_{i}, a b}^{\left(u_{v}\right)}(a, b \in\{\operatorname{Vor} H\}, i=1, \ldots, J ; v=1, \ldots, M)$ in the matrix $\mathbf{H}_{2 J \times 2 M}$ represents channel coefficients from $M$ SMTs to satellite in $J$ slots, which are independent with each other. The $h_{j_{i}, a b}^{\left(u_{v}\right)}$ is non-zero as the SMT $u_{v}(v=1, \ldots, M)$ transmits the packets in the slot $j_{i}$, otherwise, element is zero, so the $\mathbf{H}_{2 J \times 2 M}$ is sparse matrixes. It is worth noting that there must be $2 J \geq M$, otherwise, it is not possible to decode the signals of the collided SMTs. The row vectors in $\mathbf{H}_{2 J \times 2 M}$ are uncorrelated with each other due to that their channel coefficients are independent. The condition number of the $\mathbf{H}_{2 J \times 2 M}$ is low as that in [10]. Thus, the linear equations can be solved by MIMO detection algorithm. Fig. 3 gives an example of the decoded patterns of packet collisions. For the case 1 and case 2, there are only one packet and two packets in the slot \#1 and \#2, respectively. For the case 3 and case 4, there are four SMTs transmitted packet on two antennas in the slot \#3 and \#4, and slot \#5and \#6, respectively. Take the case 4 in Fig. 3 as an example, it is worth noting that there is $2 \times 2 \geq 4$, it satisfies the condition $2 J>M$, the row vectors in $\mathbf{H}_{4 \times 8}$ are uncorrelated with each other due to that their channel coefficients are independent. So it is possible to decode the signals of the collided packets by constructing a four dimension virtual MIMO signal model. The minimum mean square error (MMSE) equalization can be utilized to decode these overlapped packets. Similarly, all packets in case 1 , 2, 3 and 4 can be successfully decoded by utilizing MIMO detector or virtual MIMO detection algorithms. 


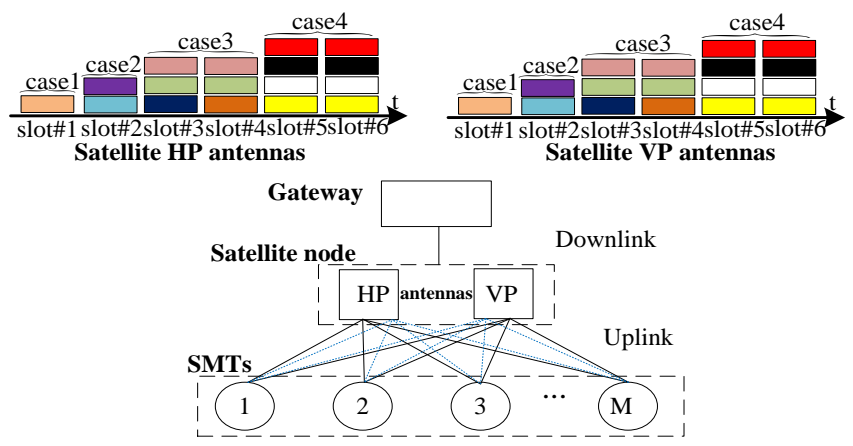

Fig. 3. The signal model of polarized MIMO

\section{The Proposed Scheme}

In the transmitter, packet replicas are transmitted with the cross polarized antennas with the degree distribution. In the receiver, one and two packets are successfully decoded by dual-polarization MIMO detection algorithms; and three or more packets can be decoded by virtual MIMO detection algorithms. The method to resolve collisions can be employed in RA systems with IRSA protocol to improve the throughput, which is dubbed as PM-IRSA. The change in packet decoding method will affect the degree distribution of IRSA, and the degree distribution should be further optimized. In this section, we first present the PM-IRSA transmitter, then give the PM-IRSA receiver.

\subsection{PM-IRSA Transmitter}

Each packet of the $u^{\text {th }}$ activated SMT contains $L_{\text {pre }}^{R A}$ preambles, $L_{\text {pay }}^{R A}$ payload symbols, $L_{\text {pos }}^{R A}$ postamble symbols, and $L_{\text {pilot }}^{R A}$ pilot symbols respectively, which can be represented as $\mathbf{x}^{(u)}=\left[\operatorname{Pre}(1), \ldots, \operatorname{Pre}\left(L_{\text {pre }}^{R A}\right), \mathbf{s}(1), \ldots, \operatorname{Pilot}(1), \ldots, \operatorname{Pilot}\left(L_{\text {pilot }}^{\text {RA }}\right), \ldots, \mathbf{s}\left(L_{\text {pay }}^{\text {RA }}\right), \operatorname{Pos}(1), \ldots, \operatorname{Pos}\left(L_{\text {pos }}^{\text {RA }}\right)\right]$. Zadaoff-Chu sequences are selected as preambles set, which are used to estimate channel parameters and recognize SMTs packets. Each activated SMT accesses the satellite node with IRSA protocol, and generates $l$ packet replicas according to degree distribution, and randomly chooses a preamble from the preamble set. Then, they are transmitted with the cross polarized antennas on $l$ slots within a TDMA frame. Each packet replica is equipped with a pointer which is utilized to indicate slot index of its replicas.

\subsection{PM-IRSA Receiver}

The satellite node receives two polarized signals with VP and HP antennas, and forwards them to the gateway station, which receives signals and stores in the memory. Firstly, preamble detection is executed by correlating local all preambles in preamble set with the received preambles signal in every slot within the received frame. A preamble is deemed as active if the correlative value is greater than a predefined threshold $\gamma_{\text {pre }}$. With the assistance of preambles detector, the number of SMTs and corresponding channel coefficients in each slot can be estimated. Then, the received packets can be decoded and some collisions can also be resolved. The packet detection process in PM-IRSA can be divided into two cases. 


\subsubsection{Single-slot Detection}

According to the system model with VP/HP polarized antennas, when one packet or two collision packets are in a slot, packets can be decoded. If a singleton packet is in a slot, a maximal ratio combining (MRC) technique can be used to combine the received signals from VP and HP antennas, then the combined signal of the packet is decoded. When two packets in a slot, the maximum likelihood (ML) MIMO detection algorithm can be used to detect the signals from two different SMTs from VP and HP antennas in the slot $i^{\text {th }}$. In this case, there are packets of two SMTs $(M=2)$ are distributed on one slots $(J=1)$, so matrix $\mathbf{H}_{2 J \times 2 M}$ represents channel coefficients from $M$ SMTs to satellite in $J$ slots, that is $\hat{\mathbf{H}}_{2 \times 2}$. It can be represented as

$$
\hat{\mathbf{x}}_{i}(k)=\underset{\mathbf{x}_{2 \times 1}(k)}{\arg \min }\left\|\mathbf{Y}_{2 \times 1}(k)-\hat{\mathbf{H}}_{2 \times 2} \mathbf{x}_{2 \times 1}\right\|^{2}
$$

where $\mathbf{x}_{2 \times 1}$ and $\mathbf{Y}_{2 \times 1}$ denote that two packets generated by the two SMTs and received signals in the one slot, respectively. Then the decoded packet replicas can be re-encoded, re-modulated, and their interferences are cancelled from all slots in locative frame by utilizing IC technique. The MIMO detection and IC are iteratively executed until none of replicas are detected.

\subsubsection{Multiple-slots Detection}

After the singleton slot detection, the number of packets in the remaining $p$ slots is more than two. The combination of $q$ slots that contain signals from $U$ SMTs may be found from remaining $p$ slots, if $U \leq 2 q$, then $2 q$ signals in $q$ slots construct a $U$ dimension virtual MIMO signal model. The minimum mean square error (MMSE) equalization can be utilized to decode these overlapped packets as that in [11]. The equalized vector can be represented as

$$
\begin{aligned}
\hat{\mathbf{x}}_{k}^{U} & =\left[\hat{X}^{\left(\hat{u}_{1}\right)}(k), \hat{X}^{\left(\hat{u}_{2}\right)}(k), \ldots, \hat{X}^{\left(\hat{u}_{U}\right)}(k)\right]^{T} \\
& =\left[\left(\hat{\mathbf{H}}_{2 q \times 2 U}\right)^{H} \hat{\mathbf{H}}_{2 q \times 2 U}+\sigma \mathbf{I}\right]^{-1}\left(\hat{\mathbf{H}}_{2 q \times 2 U}\right)^{H} \mathbf{Y}_{2 q \times 1}^{\prime}(k)
\end{aligned}
$$

where $\hat{\mathbf{H}}_{2 q \times 2 U}$ and $\mathbf{Y}_{2 q \times 1}^{\prime}$ are the estimate of the channel matrix and received signals in the virtual MIMO with $U$ SMTs in $q$ slots. If all the $q$ slots MIMO models are detected, then increase $q$ by 1 and continue to search and detect. The value of $q$ can be from 2 to $q_{\max }$. The decoded packets then re-encoded, re-modulated and their interferences are cancelled from remained slots by employing IC technique. The virtual MIMO detection is iteratively carried out as the above until the maximum number of iterations is reached.

\section{Density Evolution Analysis and Degree Distribution Optimization}

The packet loss rate of the proposed scheme is firstly derived, then the degree distribution is further optimized based on the derived result.

\subsection{Density Evolution Analysis}

The packet loss rate of scheme can be derived by means of the DE analysis. In [4], by 
introducing a graph representation of the IC process, the iterative IC convergence and PLR performance can be derived by means of the graph-based DE analysis. Literature [5] also show that the PLR of IRSA with transmit power diversity by introducing DE analysis with directly decoded probability. According to the results in [4]-[5], let $\lambda_{d}$ denote the probability that an edge is combined to a degree- $d$ packet nodes (PN), which can be computed as

$$
\lambda_{d}=\frac{d \Lambda_{d}}{\sum_{d} d \Lambda_{d}}
$$

where $\Lambda_{d}$ denotes the probability that a SMT transmits $d$ packet replicas within a frame. The edge degree distribution of PN can be expressed as $\lambda(x)=\sum_{d} \lambda_{d} x^{d-1}$. Denoted by $\rho_{d}$ the probability that an edge is combined to degree- $d$ slot nodes (SN), which be computed as

$$
\rho_{d}=\frac{d \Psi_{d}}{\sum_{d} d \Psi_{d}},
$$

where $\Psi_{d}=\left(G \Lambda^{\prime}(1)\right)^{d} e^{-G \Lambda^{\prime}(1)} / d$ ! denotes the probability that an SN has degree $d . Q_{i}$ and $P_{i}$ denote the probability that a packet cannot be removed through its replicas and directly recovered in the $i^{\text {th }}$ iteration, respectively.

The DE analysis is carried out in the following. Denote $\varphi\left(r, r_{\max }, q_{\max }\right)$ as the probability that a packet can be recovered directly in the case of $r$ collided bursts in a slot. In the proposed scheme, we assume that the maximally permissible number of packets collisions in a slot is $r_{\max }$, the maximum allowable number of slots for multiple slots detection is $q_{\max }$. According to the tree analysis in [12], we can get

$$
\begin{gathered}
Q_{i+1}=\sum_{d} \lambda_{d} P_{i}^{d-1}=\lambda\left(P_{i}\right), \\
P_{i}=1-\sum_{l=1}^{\infty} \rho_{l} \sum_{r=1}^{l} \varphi\left(r, r_{\max }, q_{\max }\right)\left(\begin{array}{l}
l-1 \\
r-1
\end{array}\right)\left(1-Q_{i}\right)^{l-r}\left(Q_{i}\right)^{r-1} \\
=1-e^{-Q_{i} G \Lambda^{\prime}(1)} \cdot \sum_{r=1}^{\infty} \frac{\left(Q_{i} G \Lambda^{\prime}(1)\right)^{r-1}}{(r-1)} \varphi\left(r, r_{\max }, q_{\max }\right) .
\end{gathered}
$$

Theoretically, when $r \leq r_{\max }, r$ collided packets can be successfully decoded directly by using the single slot detection. When $r_{\max }<r \leq q_{\max }$, if all packets of $r$ SMTs can construct a multi-slot multi-user polarized virtual MIMO signal model, these collided packets can be successfully decoded directly by using the multiple slots detection. When $r>2 q_{\max }$, all $r$ packets cannot be successfully recovered due to severe interference. Thus, the probability can be expressed as

$$
\varphi\left(r, r_{\max }, q_{\max }\right)=\left\{\begin{array}{c}
1, \quad \text { when } r \leq r_{\max } \\
\lceil r\rceil / 2(N)^{r}, \quad \text { when } r_{\max }<r \leq 2 q_{\max } \\
0, \quad \text { when } r>2 q_{\max }
\end{array}\right.
$$

When $N \rightarrow \infty$, it would be no loop in the graph, so $\lceil r\rceil / 2(N)^{r} \rightarrow 0$. By (9), (10), (11), the DE recursion for probability $p_{i}$ can be expressed as 


$$
\begin{aligned}
P_{i+1} & =\left\{\begin{array}{c}
1-e^{-\lambda\left(P_{i}\right) G \Lambda^{\prime}(1)} \cdot \sum_{r=1}^{r_{\max }} \frac{\left(\lambda\left(P_{i}\right) G \Lambda^{\prime}(1)\right)^{r-1}}{(r-1)} \text {, when } r \leq r_{\max } \\
0, \quad \text { when } r>r_{\max }
\end{array}\right. \\
& =f\left(P_{i}, G,\left\{\Lambda_{l}\right\}, r_{\max }, q_{\max }\right) .
\end{aligned}
$$

In the initial setting, $q_{0}$ is set to be 1 , and $p_{0}$ can be obtained by using (10). Combining $p_{0}$ into (12), after a maximum number of iterations $I_{\max }$, the $\mathrm{DE}$ can be expressed as $f\left(p_{I}, G,\left\{\Lambda_{d}\right\}, r_{\max }, q_{\max }\right)$. The PLR is finally obtained as

$$
\operatorname{PLR}\left(G,\left\{\Lambda_{l}\right\}, r_{\max }, q_{\max }\right)=\sum_{d} \Lambda_{d}\left(f\left(P_{I_{\max }}, G,\left\{\Lambda_{d}\right\}, r_{\max }, q_{\max }\right)\right)^{d}
$$

Based on the derived results, the throughput $T=G\left[1-P L R\left(G,\left\{\Lambda_{d}\right\}, r_{\max }, q_{\max }\right)\right]$ can be maximized by optimizing the degree distribution $\left\{\Lambda_{d}\right\}$.

\subsection{Degree Distribution Optimization}

In the proposed PM-IRSA scheme, the decoding patterns of packet collisions are different with those in the conventional IRSA scheme. The user degree distribution of the conventional IRSA scheme is no longer optimal for the PM-IRSA scheme. In order to obtain the optimal user degree distribution, which can be expressed as

$$
\begin{aligned}
\underset{\substack{\left\{\Lambda_{d}\right\}_{d \operatorname{lax}}^{d_{d=2}} \\
\text { s.t. }}}{\operatorname{maximize}} & T=G\left[1-P L R\left(G,\left\{\Lambda_{d}\right\}_{d=2}^{d_{\max }}, r_{\max }, q_{\max }\right)\right] \\
& P L R\left(G,\left\{\Lambda_{d}\right\}_{d=2}^{d_{\max }}, r_{\max }, q_{\max }\right) \leq \overline{P L R} \\
& \sum_{d=2}^{d_{\max }} \Lambda_{d}=1 \\
& 0 \leq \Lambda_{d} \leq 1, \forall d \in\left[2, \ldots, d_{\max }\right],
\end{aligned}
$$

where the first restraint restrict the relationship between throughput and PLR, and PLR depends on $G,\left\{\Lambda_{d}\right\}, r_{\max }$ and $q_{\max }$; the second constraint decides an PLR upper bound $\overline{P L R}$; the third restraint determines that the sum of all transmit packet probability levels is equal to one; the fourth constraint determines the range of $\Lambda_{d}$ and $d$. The problem (15) can be directly resolved by utilizing the differential evolution algorithm [13], which is also utilized in many existing literature, such as [4], [5]. Note that the optimal degree distribution can bring about the throughput maximization.

\section{Further Discussions}

\subsection{Computational Complexity}

As the MIMO detection algorithms and SIC iteration process are simultaneously utilized, the processing complexity at the receiver in the proposed scheme is indeed higher than that of the traditional schemes, such as PMSA, CRDSA-likes schemes, which only use one of these techniques. Similar to traditional scheme, the satellite just forwards the packets of satellite terminals to the gateway station and all the decoding operations are carried out at the gateway 
station on the ground. In this case, the overload and computational complexity of satellite can be greatly reduced. Thus the proposed scheme can be suitable to the satellite network by considering the high-performance gateway demodulator.

\subsection{Energy Efficiency}

Due to most IoT devices are powered by batteries or solar energy, the energy efficiency of satellite RA system is important, which can be denoted as [14]

$$
\psi_{E}=\frac{T(G) R_{c}}{\bar{N}_{\text {rep }} \lambda \bar{P}_{f}}
$$

where $\bar{N}_{\text {rep }}$ denotes the average number of packet replicas in a frame, $\bar{P}_{f}$ is the average transmission power per packet replica in a frame, $R_{c}$ is signaling rate and $R_{c}=R_{b} G_{g p}$, where $R_{b}$ is effective data bit rate. The random access system is usually operated at $P L R \leq 10^{-2}$ in practice to reduce the probability of retransmission and delay. Therefore, the average load $G^{*}$ corresponding to a $P L R^{*}=10^{-3}$ is considered. So the normalized throughput is $T\left(G^{*}\right)=G^{*} \cdot\left[1-P L R^{*}\left(G^{*}\right)\right] \approx G^{*}$ for the selected $P L R^{*}$ value. By utilize previous approximations, the energy efficiency can be represented as

$$
\psi_{E} \approx \frac{R \log _{2} O}{\bar{N}_{\text {rep }} N \bar{P}_{f}} .
$$

The average number of packet replicas $\bar{N}_{\text {rep }}$ of the CRDSA, CRDSA-3, IRSA with $\Lambda_{2}(x)=0.5 x^{2}+0.28 x^{3}+0.22 x^{8} \quad$ [4], NOMA-IRSA with power level $L=3$ and $\Lambda_{3}(x)=0.74 x^{2}+0.09 x^{3}+0.02 x^{4}+0.15 x^{8}$ [5], and the proposed PM-IRSA scheme with optimal degree distribution $\Lambda_{1}(x)$, are $2,3, \Lambda_{2}^{\prime}(1) \approx 3.6, \Lambda_{2}^{\prime}(1) \approx 3.03$, and $\Lambda_{1}^{\prime}(1) \approx 2.85$ respectively. CRDSA, CRDSA-3, IRSA and PM-IRSA have same the average transmission power per packet $\bar{P}_{f}$, i.e., $10^{3 / 10}$, however, the average transmission power per packet of NOMA-IRSA is $10^{(2+6+18) / 10 / 3}$. In line with [14], the energy efficiency of CRDSA, CRDSA-3, IRSA, NOMA-IRSA, and PM-IRSA system with $N=200$ slots is $1.3 \times 10^{-3}$, $8.35 \times 10^{-4}, 6.96 \times 10^{-4}, 2.24 \times 10^{-4}$ and $8.79 \times 10^{-4}$ respectively. It indicates PM-IRSA RA system has slightly lower energy efficiency compared to the CRDSA, but has higher energy efficiency compared to the CRDSA-3, IRSA and NOMA-IRSA.

\section{Simulation Results}

PM-IRSA is investigated by using the computer simulation based on MATLAB platform. The maximum allowable number of degree $d_{\max }$ and combination slots $q_{\max }$ can be fixed to 8 and 10 respectively, and $r_{\max }$ equals to 2 for practical implementation consideration. The altitude of satellite is set to be $780 \mathrm{~km}$. The carrier frequency and bandwidth are set at $2 \mathrm{GHz}$ and 20 $\mathrm{MHz}$ respectively. All schemes are simulated under the same total transmit power for each SMT for comparison fairness. The maximum number of SIC iterations $I_{\max }$ is 15 , which is the same to CRDSA/IRSA scheme. The predefined preamble detection threshold $\gamma_{p r e}$ is 3.2, which is determined by a false alarm probability [15]. The detailed parameters can be shown in Table 1. 
Table 1. Simulation Parameters

\begin{tabular}{|c|c|}
\hline Parameters & Values \\
\hline \hline The number of slot in a frame, $N$ & 200,1000 \\
\hline Traffic model & Poisson model \\
\hline Channel code and modulation model & $1 / 2$ Turbo QPSK \\
\hline Channel model & ITU-RM 1225 \\
\hline Rician factor & $10 \mathrm{~dB}$ \\
\hline The maximum number of iterations, $I_{\max }$ & 15 \\
\hline Threshold of preamble detection, $\gamma_{p r e}$ & 3.2 \\
\hline
\end{tabular}

By using the differential evolution based optimization algorithm, we can obtain the optimal degree distribution of the proposed PM-IRSA protocol, which is represented as $\Lambda_{1}(x)=0.841 x^{2}+0.021 x^{3}+0.138 x^{8}$. The performance of Polarized MIMO slotted ALOHA (PMSA) [16], CRDSA [2], CRDSA-3 [3], IRSA with $\Lambda_{2}(x)=0.5 x^{2}+0.28 x^{3}+0.22 x^{8}$ [4], NOMA-IRSA with the number of power level $L=3$ and $\Lambda_{3}(x)=0.74 x^{2}+0.09 x^{3}+0.02 x^{4}+0.15 x^{8}$ [5], PM-IRSA with $\Lambda_{2}(x)$ and PM-IRSA with $\Lambda_{1}(x)$ and $N=200,1000$ are compared and investigated.

Fig. 4 shows the number of users in a slot versus the probability of miss-detection and the mean square error (MSE) of channel estimation at different preamble length $L_{p r e}^{R A}$. We can also see that, in the range of 2 to 6 users in a slot, the probabilities of miss-detection and the MSEs of channel estimation are less than $10^{-2}$ when $L_{\text {pre }}^{R A}=382$.

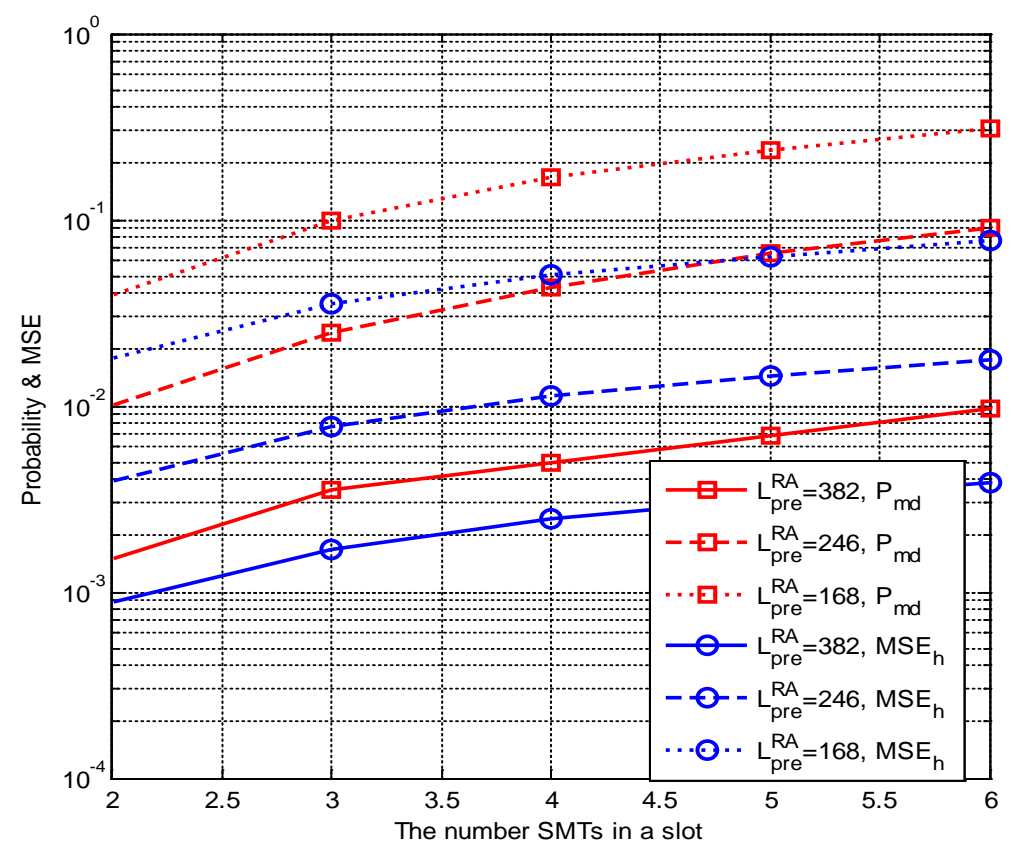

Fig. 4. The probability of miss-detection and MSE of channel estimation vs. the number of users in a slot. 


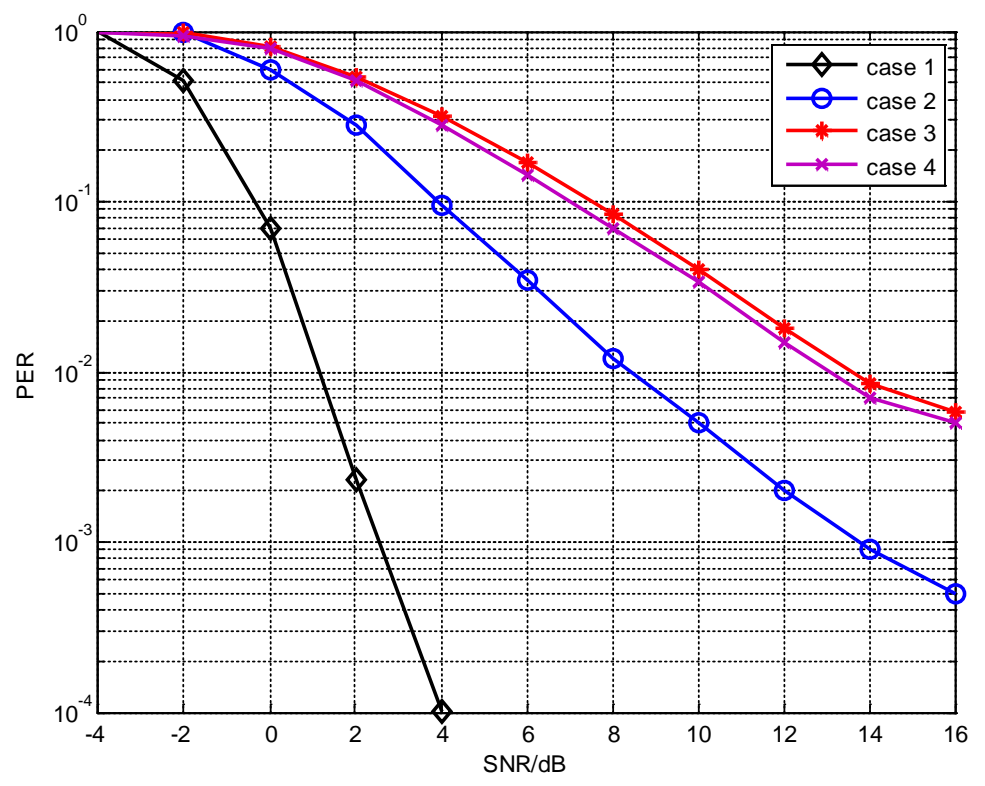

Fig. 5. PER versus SNR for case 1, case 2, case 3 and case 4.

We can see from Fig. 5 that the packet error rate (PER) for the case 1, 2, 3 and 4 in PM-IRSA, corresponding to Fig. 3, which denote the cases of one packet in a given slot, two packets in a given slot, six packets and eight packets of four users in two given slots, respectively. It can be seen that the PER are below $10^{-1}$ when the SNR are larger than $0 \mathrm{~dB}$, $4 \mathrm{~dB}, 8 \mathrm{~dB}$ and $7 \mathrm{~dB}$ for the case $1,2,3$ and 4 , respectively, which shows that all packets for case 1, 2, 3 and 4 can be effectively decoded with MIMO detection.

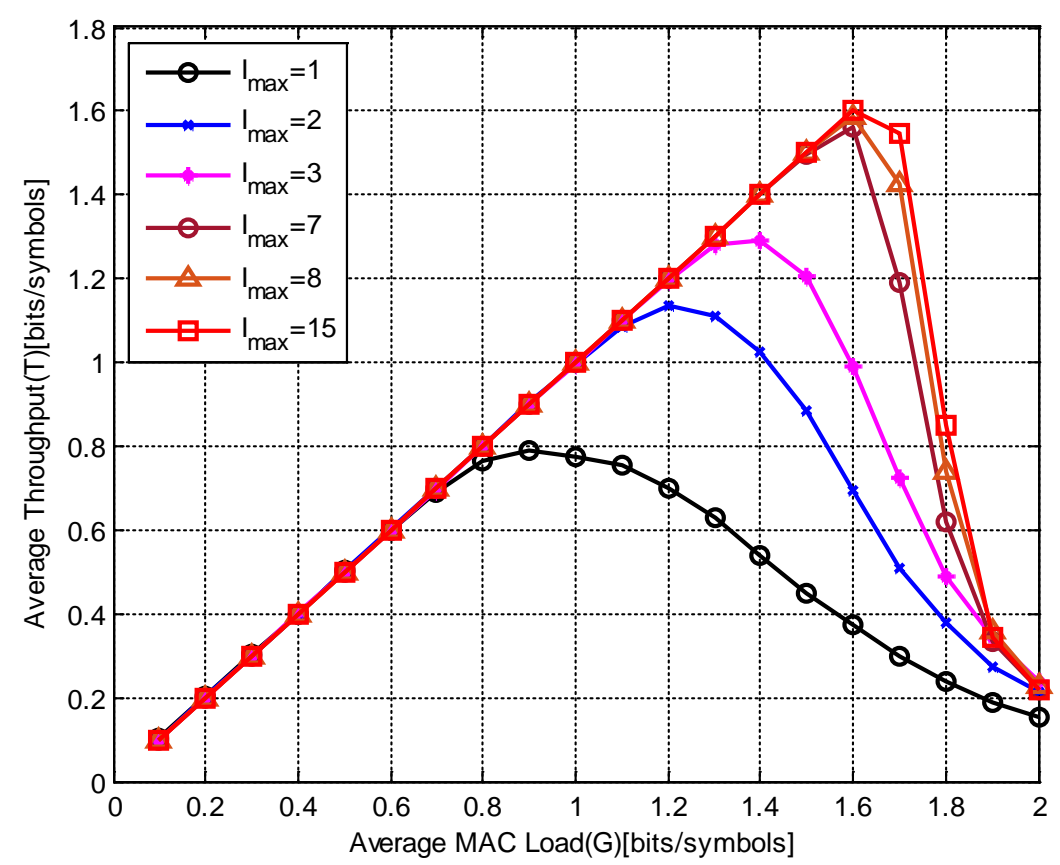

Fig. 6. Normalized throughput versus normalized load at different iterative times. 
Fig. 6 shows the throughput performance of PM-IRSA versus load at different maximum iterative times $I_{\max }$. We can see how many iterations of IC processing can cause the normalized throughput to converge. From Fig. 6, it can be seen that the throughput improvement is gradually decreasing as the $I_{\max }$ increases. The random access system appear to achieve the convergence when maximum iterative time equals to $I_{\max }=15$.

For throughput and PLR simulation, the SNR is greater than $10 \mathrm{~dB}$. From Fig. 7, it can be seen that the peak normalized throughput of PM-IRSA with optimal degree distribution $\Lambda_{1}(x)$, $N=200$ can reach about 1.6 bits/symbol, which is $90.5 \%$ larger than that of PMSA, 205.3\% larger than that of CRDSA, 138.8\% larger than that of CRDSA-3, 128.6\% larger than that of IRSA, $11.1 \%$ larger than that of NOMA-IRSA with $L=3,15.9 \%$ larger than that of PM-IRSA with original $\Lambda_{2}(x)$, respectively. In addition, it can be seen that the linear region of PM-IRSA is enlarged compared with that of PMSA and IRSA, which achieves linear throughput up to a normalized load of 1.5 bits/symbol with the $P L R \leq 10^{-2}$. As the frame length increases, the throughput of the PM-IRSA also increases and approaches its asymptotic theoretical one.

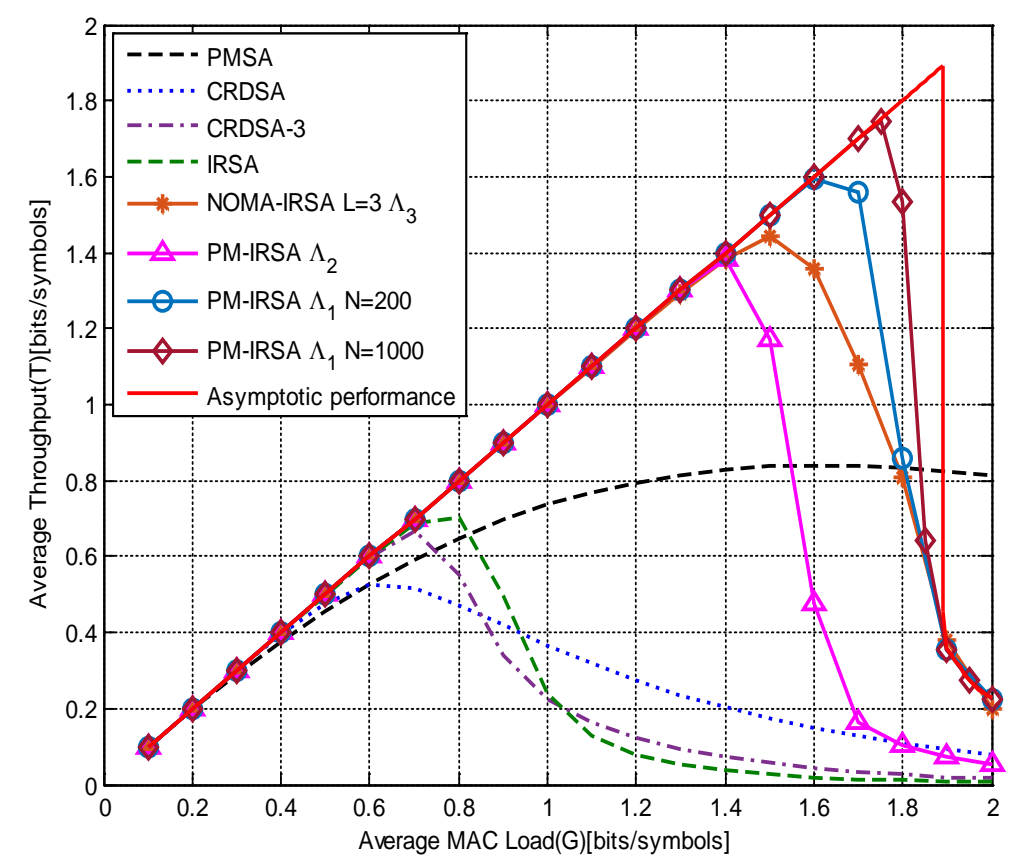

Fig. 7. Throughput vs. the normalized load.

We can see from Fig. 8 that packet loss ratio of PM-IRSA with optimal degree distribution $\Lambda_{1}(x)$ is much better than those of all given schemes, especially in the case of overload. When the load is between 1 and 1.4, the PLRs of CRDSA, CRDSA-3 and IRSA are all close to 1 , that is, all packets are lost, while the PLR of PM-IRSA with $\Lambda_{1}(x)$ is below $10^{-2}$. In addition, the PLR of PM-IRSA with $\Lambda_{1}(x)$ is better than NOMA-IRSA with $L=3$ under high overload. As the frame length increases, the PLR of the PM-IRSA with $\Lambda_{1}(x)$ become steeper and approaches its asymptotic theoretical PLR, which validates our analysis. 


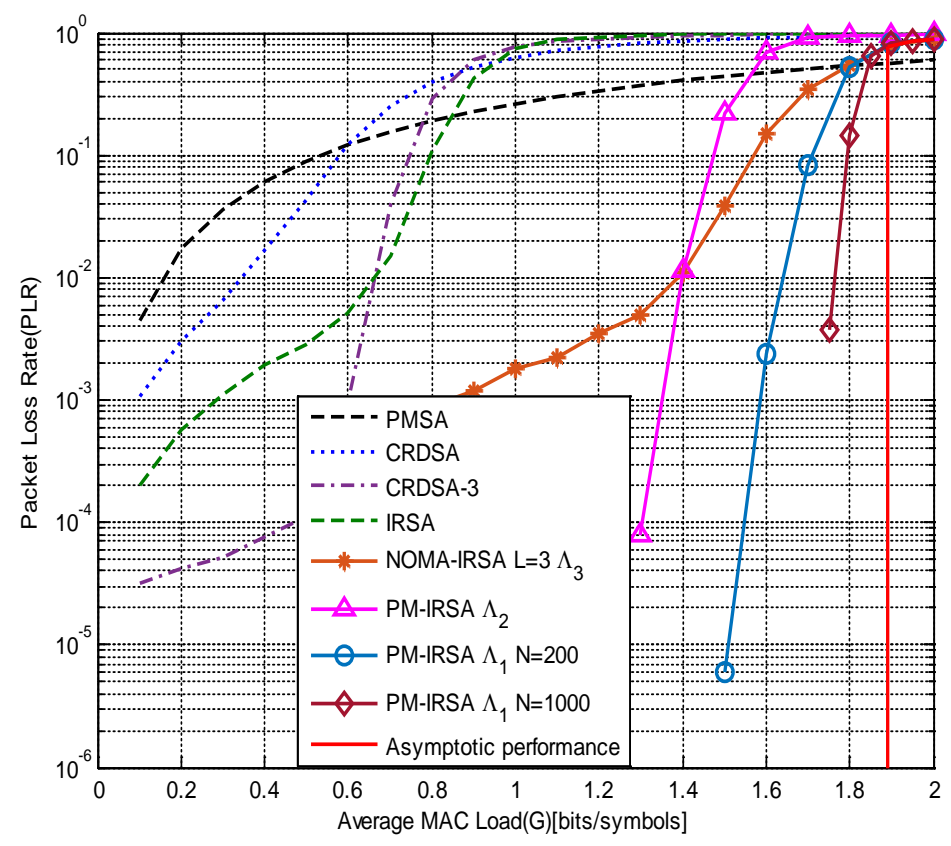

Fig. 8. Packet loss ratio vs. the normalized load.

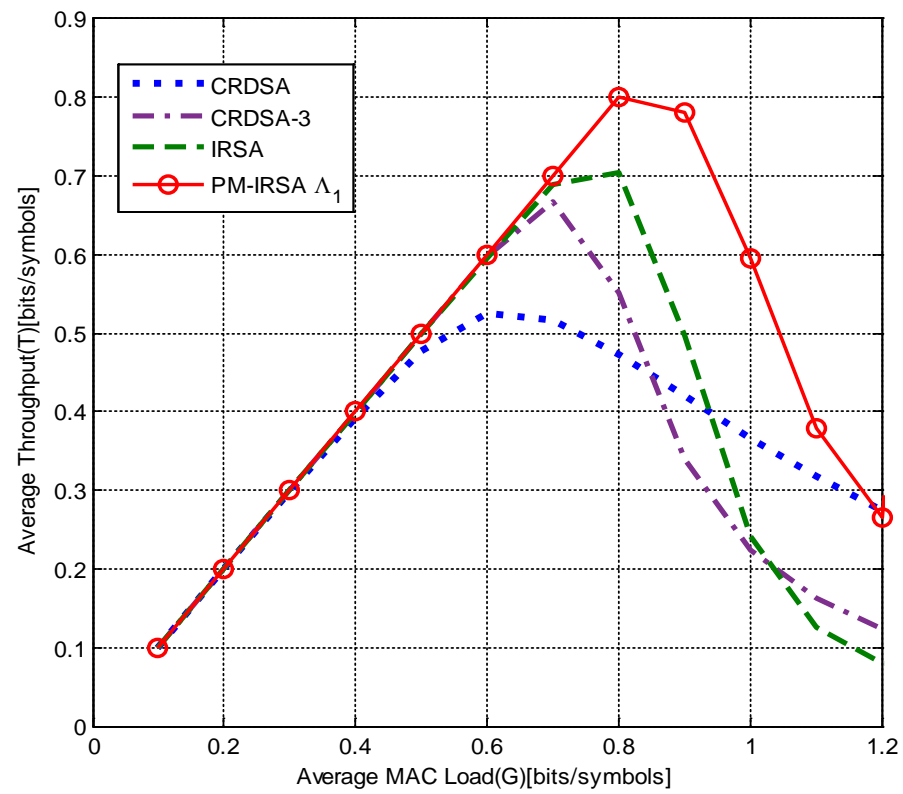

Fig. 9. Throughput of single channel vs. the normalized load.

In Fig. 9 show that the performance comparison of single channel which be easily obtained by the performance of two polarized channels, that is, the performance of single channel can be obtained by normalizing the throughput with the number of channels. The throughput of 
CRDSA, CRDSA-3 and IRSA with $\Lambda_{2}(x)$ are used as comparisons with the proposed PM-IRSA with $\Lambda_{1}(x)$. In Fig. 9 show that the single channel peak throughput of PM-IRSA with optimal degree distribution $\Lambda_{1}(x), N=200$, can reach about $0.8 \mathrm{bits} / \mathrm{symbol}$, which is 53.8\% larger than that of CRDSA, 21.3\% larger than that of CRDSA-3, respectively. In addition, the linear throughput region of PM-IRSA is extended.

\section{Conclution}

PM-IRSA protocol with multiple packet detection capability has proposed in this paper, which employs polarization characteristic of satellite link and MIMO detection to decode multiple collided packets. The PLR of the PM-IRSA in the asymptotic regime is derived by resorting to the DE analysis. Based on this derived results, user degree distribution is optimized by means of the differential evolution based optimization algorithm, which maximizes the throughput. PM-IRSA protocol can overcome the bottleneck of traditional protocols and greatly outperform traditional schemes.

\section{References}

[1] M. D. Sanctis, "Satellite communications supporting Internet of Remote Things," IEEE Internet of Things Journal, vol. 3, no. 1, pp. 113-123, Feb. 2016. Article (CrossRef Link)

[2] E. Casini, R. De Gaudenzi, and O. del Rio Herrero, "Contention resolution diversity slotted ALOHA(CRDSA):An enhanced random access scheme for satellite access packet networks," IEEE Transactions on Wireless Communication, vol. 6, no. 4, pp. 1408-1419, Apr. 2007. Article (CrossRef Link)

[3] O. del Rio Herrero and R. De Gaudenzi, "A high-performance MAC protocol for consumer broadband satellite systems," in Proc. of IET AIAA International Communication, 2009. Article (CrossRef Link)

[4] G.Liva, "Graph-based analysis and optimization of contention resolution diversity slotted ALOHA," IEEE Transactions on Communications, vol. 59, no. 2, pp. 477-487, Feb.2011. Article (CrossRef Link)

[5] X. Shao, Z. Sun, M. Yang, et al, "NOMA-Based Irregular Repetition Slotted ALOHA for Satellite Networks," IEEE Communications Letters, vol. 23, no. 4, pp. 624-627, Apr 2019.

Article (CrossRef Link)

[6] M. Bacco, P. Cassarà, E. Ferro, et al, "Generalized encoding CRDSA: Maximizing throughput in enhanced random access schemes for satellite," in Proc. of International Conference on Personal Satellite Services. Springer, pp. 115-122, 2013. Article (CrossRef Link)

[7] A. Mengali, R. De Gaudenzi, and P. D. Arapoglou, "Enhancing the physical layer of contention resolution diversity slotted ALOHA," IEEE Transactions on Communications, vol. 65, no. 10, pp. 4295-4308, 2017. Article (CrossRef Link)

[8] F. Clazzer, E. Paolini, I. Mambelli, et. al, "Irregular repetition slotted ALOHA over the Rayleigh block fading channel with capture," in Proc. of IEEE International Conference on Communications (ICC), pp. 1-6, 2017. Article (CrossRef Link)

[9] E. Nisioti and N. Thomos, "Fast Q-learning for Improved Finite Length Performance of Irregular Repetition Slotted ALOHA," IEEE Transactions on Cognitive Communications and Networking, vol. 6, no. 2, pp. 844-857, 2020. Article (CrossRef Link)

[10] Q. H. Abbasi, H. E. Sallabi, E. Serpedin, et al, "Condition number variability of ultra wideband MIMO on body channels," in Proc. of IEEE Intern Works Anten Techn(IWAT), pp.167 - 169, Mar. 2016. Article (CrossRef Link) 
[11] B. Zhao, G. Ren, and H. Zhang, "Pre-Weighting based Contention Resolution Diversity Slotted ALOHA Scheme for Geostationary Earth Orbit Satellite Networks," IEICE Transactions. on Communications, E102-B(3), 648-658, 2019. Article (CrossRef Link)

[12] M. Luby, M. Mitzenmacher and A. Shokrollahi, "Analysis of random processes via and-or tree evaluation," in Proc. of 9th Annual ACM-SIAM Symp. Discrete Algorithms, San Francisco, CA, USA, Jan. 1998.

[13] R. Storn and K. Price, "Differential evolution-a simple and efficient heuristic for global optimization over continuous spaces," J. Global Optimization, vol. 11, no. 4, pp. 341-359, Dec. 1997. Article (CrossRef Link)

[14] A. Mengali, R. De Gaudenzi, C. Stefanovic, “On the Modeling and Performance Assessment of Random Access with SIC,” IEEE Journal on Selected Areas in Communications, vol. 36, no. 2, pp. 292-303, 2018. Article (CrossRef Link)

[15] Q. Wang, G. Ren, J. Ying, “A Multiuser Detection Algorithm for Random Access Procedure with the Presence of Carrier Frequency Offset in LTE Systems," IEEE Transactions on Communications, vol. 63, no. 9, pp.3299-3312, 2015. Article (CrossRef Link)

[16] J. Bai and G. Ren, "Polarized MIMO Slotted ALOHA Random Access Scheme in Satellite Network," IEEE Access, vol.5, pp. 26354-26363, Nov. 2017. Article (CrossRef Link) 


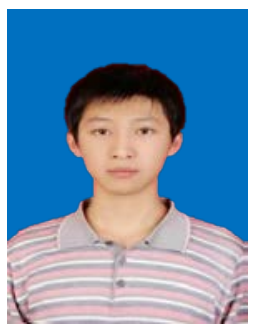

Jingrui Su was born in Gansu, China, in 1990. He is currently pursuing the Ph.D. degree in communication and information systems with Xidian University, Xi'an, China. His research interests include random access technologies and M2M satellite networks.

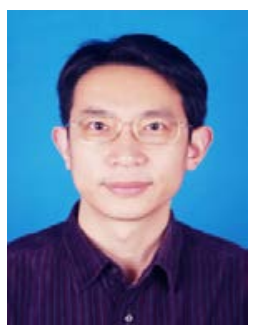

Guangliang Ren (M’06) was born in Jiangsu, China, in 1971. He received the B.S. degree in communications engineering from Xidian University, Xian, China, in 1993, the M.S. degree in signal processing from the Academy of China Ordnance, Beijing, China, in 1996, and the Ph.D. degree in communications and information systems from Xidian University, in 2006. He is currently a Professor with the School of Telecommunications Engineering, Xidian University. He is the author of more than 40 research papers in journals and conference proceedings, such as IEEE Transactions on Wireless Communications, IEEE Transactions on Communications, IEEE Transactions on Vehicular Technology and an author or coauthor of three books. His research interests include wireless communications and digital signal processing, particularly MIMO systems, WiMax, LTE, etc.

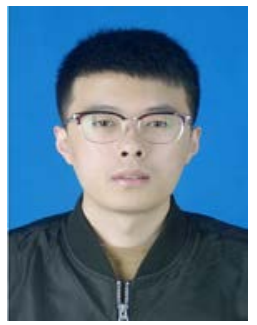

Bo Zhao was born in Shandong, China, in 1992. He is currently pursuing the Ph.D. degree in communications and information systems with Xidian University, Xi'an, China. His research interests include random access technologies and multiple-input multiple-output systems.

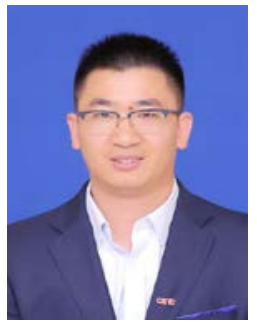

Jian Ding was born in Henan, China, in 1987. He is currently pursuing the Ph.D. degree in communications and information systems with Xidian University, Xi'an, China. His research interests include random access technologies and M2M satellite networks. 